1 Science and Technology Institute, São Paulo State University (UNESP), São Jose dos Campos, SP, Brazil.

${ }^{2}$ Department of Dentistry,

Prosthodontics Unit, Federal University of Rio Grande do Norte (UFRN), Natal, Rio Grande do Norte, Brazil

${ }^{3}$ Department of Restorative Dentistry, Federal University of Juiz de Fora, Juiz de Fora, MG, Brazil.
Corresponding author:

Jean Soares Miranda

(iD) https://orcid.org/0000-0001-5379-0155 Address: Av Engenheiro Francisco José Longo, 777, Jardim São Dimas, São José dos Campos, São Paulo, Brazil.

Zip Code: $12245-000$

Phone: +55 32988239151

e-mail: jeansoares@msn.com

Received: January 20, 2019

Accepted: June 02, 2019

\section{Different surface treatment protocols of a Y-TZP ceramic with a superficial glaze layer}

\author{
Jean Soares Miranda ${ }^{1, *}$, Ronaldo Luís Almeida de \\ Carvalho ${ }^{1}$, Aline Serrado de Pinho Barcellos ${ }^{1}$, Rodrigo \\ Othávio Assunção e Souza², Estevão Tomomitsu \\ Kimpara ${ }^{1}$, Fabíola Pessôa Pereira Leite ${ }^{3}$
}

Aim: evaluate the influence of etching time with hydrofluoric acid on the bond strength of a Yttrium-stabilized polycrystalline tetragonal zirconia (Y-TZP) ceramic with a superficial glaze layer and a resin cement. Methods: Y-TZP blocks were cut to obtain 40 samples. They were distributed into four groups $(n=10)$ : control treated by sandblasting with silica-coated alumina (RS) and three glazed experimental groups with different etching times: GS20s, GS60s and GS100s. Cementation was done with a universal adhesive and a resin cement. Two cement cylinders were made in each block. After thermocycling, the shear bond test was performed. Two extra samples of each group were made to obtain profilometry, scanning electron microscopy, mapping and backscattered electron detector images. Energy dispersive spectrometry and goniometry were also performed Results: Kruskal-Wallis and Dunn tests demonstrated bond strength differences only between the RS (22.10MPa) and the GS groups (GS20s: 8,10Mpa; GS60s: 10.49MPa; GS100s: 7.53MPa) ( $p=0.001$ ), but there was no difference among the experimental groups ( $p>0.05$ ). The contact angles were $55.33^{\circ}(R S) ; 70.78^{\circ}$ (GS100s); $48.20^{\circ}$ (GS60s) and $28.73^{\circ}$ (GS20s). ANOVA and Tukey test demonstrated similar wettability of RS to GS60s and GS100s ( $p>0.05$ ), but all the experimental groups were statistically different between them $(p<0.001)$. Qualitative image analysis revealed an irregular glaze distribution after etching. The thickness of the remaining glaze layer measured by profilometry was $5 \pm 1 \mu \mathrm{m}$ (GS20S), $4 \pm 1 \mu \mathrm{m}$ (GS60S) and $3 \pm 1 \mu \mathrm{m}$ (GS100s). Conclusion: The etching time of glazed zirconia did not influence the adhesive strength of the ceramic to the resin cement.

Keywords: Zirconium. Glass. Dental cements. 


\section{Introduction}

Yttrium-stabilized polycrystalline tetragonal zirconia (Y-TZP) has high chemical stability, high flexural strength, radiopacity, biocompatibility and low thermal conductivity, what makes it a good material for oral rehabilitation ${ }^{1-6}$. However, the clinical success of dental ceramic prostheses also depends on a luting protocol ${ }^{1-7}$. However, this is not yet establish for zirconia8. In order to achieve a durable bond between zirconia and resin cement, surface treatments are required to this material, creating micro mechanical -retentions ${ }^{2-5,9-11}$. They are: sandblasting with aluminum oxide particles ${ }^{12}$, sandblasting with silica-coated alumina ${ }^{1,12,13}$; Er:YAG laser irradiation ${ }^{1}$ and plasma spraying ${ }^{14}$.

The sandblasting with silica-coated alumina presents good bond results ${ }^{1,12,13}$. It can chemically modify the ceramic making it reactive with the resin, creates roughness and irregularities, what increases the surface area and the wettability, allowing the cement to flow $3,5,6,9,10,15$. Nevertheless, there are concerns about this treatment. The impact of this abrasion may lead to long-term surface changes, to local silica network distortions that are not sustained, and/ or due to the emergence of a new zirconia phase, create stress and form lateral cracks ${ }^{14}$. Thus, use of alternative methods to abrasion have been suggested, such as application of a thin glaze layer and/or the use of universal primers on the zirconia surface $6,10,16$.

The surface treatment referred as vitrification involves applying a thin glass layer under the surface of the Y-TZP ceramic. This aims to enrich the surface with a vitreous material and allow the hydrofluoric acid (HF) etch of this surface, changing the topography and providing area of mechanical retention $3,5,7,17-20$. In addition, this etching would increase the ceramic surface energy and its adhesive potential, a prerequisite for a stable and durable bonding of the resin cement to the substrate $3,5,7,17-20$. However, the ideal HF etching time on this surface is not yet defined.

Following a simplified strategy, some new universal adhesives have been developed to be used with various restorative materials. They allow the bond to zirconia without the use of primers because they have silane, which promotes adherence to silica-based surfaces, and MDP (10-methacryloyloxydecyl dihydrogen phosphate), which is designed to create a chemical bond to metal oxides such as zirconium 13,10,21,22. It has been suggested that these monomers can interact with the $\mathrm{Y}$-TZP and the resin cement, enabling chemical adhesion through van der Waals forces or hydrogen bonds ${ }^{21,22}$.

Studies are being published with the intention of achieving better adhesion to dental zirco$n^{2} a^{2,3,5,9,9,10,15,21-22}$. They involve a variety of surface treatment methods, adhesion promoters or cements, but an efficient and long-lasting protocol for zirconia luting has not yet been established $^{2}$. Y-TZP vitrification followed by HF etching is a method already reported in the literature ${ }^{11,19}$. However, in a temptive to establish a luting protocol, some problems should be better studied, such as the influence of the HF etching time on this new vitreous layer. Thus, this research aimed to evaluate the influence of different etching times with $10 \%$ hydrofluoric acid on the bond strength between a vitrified Y-TZP ceramic and a resin cement. These times were chosen from the conventional 60 s etching to vitreous ceramic. The intention was also to verify if some higher or lower etching time would modify the bond strength. The null hypotheses were that the surface treatment type would not influence the bond strength and the etching time of the experimental groups would not alter this result. 


\section{MATERIALS AND METHODS}

\section{Sample preparation:}

The materials used in this study, their trademarks, manufacturers, composition and lots are presented in Table 1.

Y-TZP blocks (IPS e.max® ZirCAD - Ivoclar-Vivadent, Schaan, Liechtenstein) were cut to a standard size of $15 \times 15 \times 2 \mathrm{~mm}$ with a diamond cutting disc (Extec High Concentration, Enfield - CT, USA) in a precision cutting machine (IsoMet ${ }^{\circledR} 1000$ Precision Saw, Buehler, Lake Buff-IL, USA) to obtain 48 samples. Both sides of the samples were regularized with \# 180, \# 600 and \# 1200 granule sandpaper (Norton Saint- Gobain, São Paulo, Brazil). Prior to sintering, the samples were washed in an ultrasonic bath (Cristófoli Ultrasonic Washer, Campo Mourão, Paraná, Brazil) in isopropyl alcohol for eight minutes. The sintering was carried out in a Zyrcomat T oven (VITA, Zahnfabrick, Germany) up to the temperature of $1530^{\circ} \mathrm{C}$. After this process, the final samples dimensions were $12 \times 12 \times 1.5 \mathrm{~mm}$.

The ceramic blocks were then randomly distributed into four groups $(n=10)$ by Radom Alocater (Mads Haahr, Dublin, Irish). One was the control group, in which sandblasting with silica-coated alumina was performed by Rocatec Soft (3M ESPE, St. Paul, Minnesota, USA) (RS), and three experimental groups were vitrified with Glaze Spray VITA Akzent Plus (Vita Zanhfabrik, Bad Sachingen, Germany) on the adhesion surface and etched with 10\% HF for 20s (GS20s), 60s (GS60s) or 100s (GS100s).

The RS group had sandblasting done at a distance of $10 \mathrm{~mm}$ between the zirconia surface and the tip of the apparatus (Dento-PrepTM, RØNVIG A / S) with a $45^{\circ}$ slope, at 2.8 bars of pressure for $15 \mathrm{~s}$. In the experimental groups, the Glaze Spray VITA

Table 1. Commercial brand, use, manufacturer, composition and lot of materials used in the research.

\begin{tabular}{|c|c|c|c|c|}
\hline Brand & Material Type & Manufacturer & Composition & Lot \\
\hline $\begin{array}{l}\text { IPS e.max } \\
\text { ZirCAD }\end{array}$ & $\begin{array}{l}\text { Y-TZP } \\
\text { ceramic }\end{array}$ & $\begin{array}{l}\text { Ivoclar-Vivadent, } \\
\text { Schaan, } \\
\text { Liechtenstein }\end{array}$ & $\begin{array}{c}\mathrm{ZrO} 2+\mathrm{HfO}_{2}(94.4 \mathrm{wt} \%), \mathrm{Y}_{2} \mathrm{O}_{3}(5.2 \\
\text { wt\%), } \mathrm{Al}_{2} \mathrm{O}_{3}(0.2-0.5 \mathrm{wt} \%)\end{array}$ & M24091 \\
\hline $\begin{array}{l}\text { Rocatec }{ }^{\circledR} \\
\text { Soft }\end{array}$ & $\begin{array}{l}\text { Silica-coated } \\
\text { alumina }\end{array}$ & $\begin{array}{l}\text { 3M ESPE, St. Paul, } \\
\text { Minnesota, USA }\end{array}$ & $30 \mu \mathrm{m}$ Silica-coated alumina & 424975 \\
\hline $\begin{array}{l}\text { VITA Akzent }{ }^{\circledR} \\
\text { Plus }\end{array}$ & Glaze Spray & $\begin{array}{l}\text { Vita Zanhfabrik, Bad } \\
\text { Sachingen, Germany }\end{array}$ & 111-29-5 pentano-1,5-diol & A0764 \\
\hline $\begin{array}{l}\text { Single Bond } \\
\text { Universal }\end{array}$ & $\begin{array}{l}\text { Universal } \\
\text { adhesive }\end{array}$ & $\begin{array}{l}\text { 3M ESPE, Sumaré, } \\
\text { SP, Brazil }\end{array}$ & $\begin{array}{l}\text { MDP, Vitrebond copolymer, HEMA, } \\
\text { silane, dimethacrylate resins, fillers, } \\
\text { initiators and ethanol }\end{array}$ & 1511900505 \\
\hline $\begin{array}{l}\text { RelyX } \\
\text { Ultimate }\end{array}$ & Resin cement & $\begin{array}{l}\text { 3M ESPE, Sumaré, } \\
\text { SP, Brazil }\end{array}$ & $\begin{array}{l}\text { Base: Methacrylate monomers/ } \\
\text { radiopaque, silanated fillers, initiators, } \\
\text { stabilizers, rheological additives. } \\
\text { Catalyst: Methacrylate monomers, } \\
\text { radiopaque, alkaline fillers, initiators, } \\
\text { stabilizers, pigments, rheological } \\
\text { additives, fluorescence dye, dark } \\
\text { cure activator for Scotchbond } \\
\text { Universal adhesive. }\end{array}$ & 1509800356 \\
\hline $\begin{array}{l}\text { Condac } \\
\text { Porcelana }\end{array}$ & $\begin{array}{l}\text { Hydrofluoric } \\
\text { acid }\end{array}$ & $\begin{array}{l}\text { FGM, Pinheiros, SP, } \\
\text { Brazil }\end{array}$ & $\begin{array}{c}10 \% \mathrm{HF} \text {, water, thickener, surfactant } \\
\text { and colorant }\end{array}$ & 250215 \\
\hline
\end{tabular}


AKZENT Plus (Vita Zahnfabrik) was applied at a standard of $3 \mathrm{~cm}$ distance from the ceramic surface, taking the time required for the ceramic surface to be completely covered. Then, the samples were taken to a VITA VACUMAT 6000 MP oven (VITA, Zahnfabrik, Germany) for glaze firing process ${ }^{19}$.

\section{Sample luting:}

The experimental group samples were etched with 10\% HF (Condac Porcelana FGM, Pinheiros, SP, Brazil) at different time intervals according to the group, and washed by air-water spray for twice the HF etching time. The blocks were then cleaned again in sonic bath (Cristófoli Ultrasonic Washer) for $5 \mathrm{~min}$ in distilled water to remove the acid precipitate.

These blocks were fixed in a cylinder of acrylic resin and Single Bond Universal adhesive (3M ESPE®, St. Paul, Minnesota, USA) was applied with a microbrush (Vigodent, Rio de Janeiro, RJ, Brazil) for 60s, then left to act for 20s and light air was applied for 5s, without light curing. Soon after, two silicon transparent cylindrical matrices (Tygon tubing, TYG-030, Saint-Gobain Performance Plastic, Miami Lakes, Florida, USA) of $3 \mathrm{~mm}$ internal diameter by $3 \mathrm{~mm}$ height were placed under the samples, totaling twenty adhesive interfaces per group to be tested. Then, RelyX Ultimate dual resin cement (3M ESPE, St. Paul, Minnesota, USA) was manipulated following the manufacturer's recommendations and immediately inserted into the matrices with the aid of a centrix syringe (Polidental Ind. e Com. Ltda, São Paulo, SP, Brazil). Light curing was performed with the Radii-Cal LED (SDI, Pinheiros, SP, Brazil) with an intensity of $1200 \mathrm{~mW} / \mathrm{cm}$ and an application time of 40 s on each side ${ }^{20}$.

After luting, all samples were stored in distilled water (Olidef, Ribeirão Preto, São Paulo, Brazil) at $37^{\circ} \mathrm{C}$ for 24 hours. Next, the silicon matrices were removed with number 12 blades (Becton Dickinson, New Jersey, USA), obtaining the final specimens for the research.

\section{Shear bond strength test and failure mode analysis:}

All the specimens were subjected to aging by thermocycling for 6,000 cycles (Nova Ética, São Paulo, SP, Brazil) between two water baths of $5^{\circ} \mathrm{C}$ and $55^{\circ} \mathrm{C}$, with a time of $30 \mathrm{~s}$ each $^{3,20}$. After this, they were subjected to shear bond strength testing using a universal testing machine (EMIC, DL 2000, São José dos Pinhais, Paraná, Brazil) at a crosshead speed of $1.0 \mathrm{~mm} / \mathrm{min}$. Shear bond strength (in MPa) was calculated by dividing the load at fracture (in Newtons), with the bonding interface area $\left(28.26 \mathrm{~mm}^{2}\right)$.

For failure analysis, a Stemi 2000-C stereomicroscope (Karl Zeiss) with 16X magnification was used coupled with a digital camera. All samples were analyzed for failure type classification: adhesive (at the adhesive-ceramic interface), cohesive (involving only one of the substrates) or mixed (involves the adhesive interface and also one of the substrates).

\section{Contact Angle analysis:}

For contact angle analysis, two extra non-cemented specimens of each group were made. The contact angle was measured by a goniometer (Ramé Hart-Inc, 100-00-115, Mountain Lakes, Nova Jersey, EUA) in a controlled-temperature environment. The 
goniometer was connected to a computer equipped with specific software $(\mathrm{RHI} 2001$ Imaging Software), and the sessile drop technique was implemented. A drop of distilled water was placed on the ceramic surface using a syringe, and the contact angle was measured for 10 seconds (30 frames per second). Five measurements were performed for each sample, totaling 10 measurements per group.

\section{Surface analysis:}

One of the extra samples was also examined in a digital optical profilometer (Wyko, Modelo NT 1100, Veeco, Tucson, USA) connected to a computer with image software (Vision 32, Veeco, Tucson, USA) to perform surface micrographs (qualitative analysis of three-dimensional geometry). The glaze layer after 10\% HF etching was also measured using the profilometer, performed by four micrographs per sample. Then, the glaze layer was calculated by averaging the obtained values.

The same samples were then cleaned with 70\% alcohol (Alves Santa Cruz Ltda. - Guarulhos, São Paulo, Brazil), dried and metallized (EMITECH SC7620), receiving a thin layer $(12 \mathrm{~nm})$ of gold alloy. They were examined using a scanning electron microscope (SEM; INSPECT S50, FEl, Czech Republic) to obtain mapping, backscattered electron detector (BSE) and conventional SEM images.

Energy Dispersive Spectrometry (EDS) was then used for chemical element analysis. The readings were performed at a distance of $12 \mathrm{~mm}$ and $20 \mathrm{kV}$ accelerating voltage. The main elements were analyzed in 100 s real time for each measured area $\left(1 \mathrm{~mm}^{2}\right)$.

\section{Statistical Analysis:}

To evaluate the surface treatment influence on bond strength, the data were submitted to Kruskal-Wallis and Dunn statistical tests. In order to evaluate the influence of these different treatments on the zirconia wettability, one-way ANOVA and Tukey tests were applied. The significance level for all tests was 95\%.

\section{RESULTS}

\section{Shear bond strength test and failure mode:}

The Kruskal-Wallis test revealed a significant interaction of the surface treatment $(p=0.001)$. Using Dunn's test $(p \leq 0.05)$, it was possible to verify that bond strength mean values of the RS group were statistically higher than the GS20s, GS60s and GS100s groups. However, the etching time did not influence the bond strength of these experimental groups (Table 2). Pre-test failures occurred in GS20s (9), GS60s (14) and GS100s (16) groups. They were characterized by cement detachment during thermocycling. Stereomicroscopic analysis revealed complete adhesive failures (100\%).

\section{Contact Angle:}

The control group was similar to GS60s and GS100s. GS20s group had lower contact angle and therefore better wettability. It was observed that the lower the glaze layer etching time, the better the surface wettability. In addition, all experimental groups were statistically different from each other $(p<0.001)$ (Table 2$)$. 


\section{Surface analysis:}

Three-dimensional geometry analysis by profilometry revealed a decrease in the glaze layer thickness; it was inversely proportional to the $10 \% \mathrm{HF}$ etching time. Mean of the residual glaze layer thickness was: $5 \pm 1 \mu \mathrm{m}$ for GS20s, $4 \pm 1 \mu \mathrm{m}$ for GS60s, and $3 \pm 1 \mu \mathrm{m}$ for GS100s (Figure 1).

The SEM of the RS group showed a homogeneous surface composed by zirco-

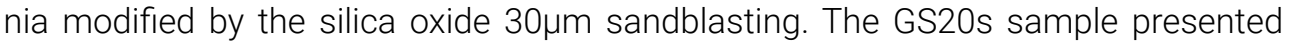

Table 2. Dunn test results for bond strength values (MPa) and contact angles results $\left(^{\circ}\right)$ analyzed by oneway ANOVA and Tukey test ( $p<0.001)$. Different letters indicate a significant difference between groups.

\begin{tabular}{lcccccc}
\hline & N & \multicolumn{2}{c}{ Bond Strength Mean (MPa) } & N & \multicolumn{2}{c}{ Contact Angle $\left(^{\circ}\right)$} \\
\hline RS & 20 & $22.10(2.78)$ & a & 10 & $55.33(10.70)$ & a b \\
\hline GS20s & 11 & $8.10(5.59)$ & b & 10 & $70.78(6.69)$ & ${ }^{\circ}$ \\
\hline GS60s & 6 & $10.49(5.38)$ & b & 10 & $48.20(10.57)$ & $\mathrm{b}$ \\
\hline GS100s & 4 & $7.53(4.62)$ & b & 10 & $28.73(19.75)$ & a \\
\hline
\end{tabular}
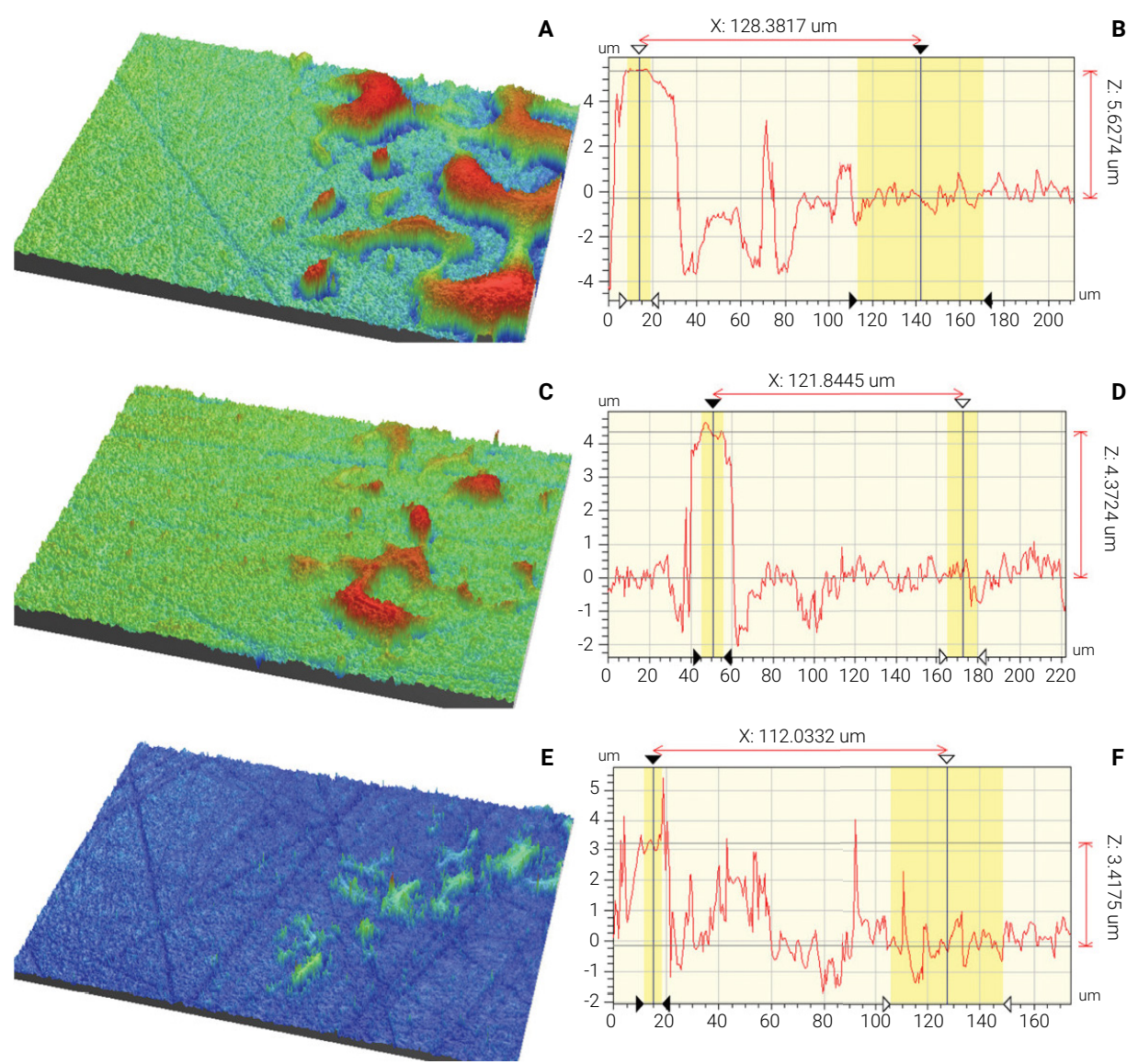

Figure 1. Profilometry 3D image of the of GS20s (A), GS60s (C) and GS100s (E) showing irregularity in the arrangement of applied glaze spray and their remaining glaze thickness measurements, indicating the average layer thickness $5 \pm 1 \mu \mathrm{m}(\mathrm{B}), 4 \pm 1 \mu \mathrm{m}(\mathrm{D})$ and $3 \pm 1 \mu \mathrm{m}(\mathrm{F})$. 
a surface predominantly composed of zirconia with few residual glaze layer areas dispersed on the ceramics ("glaze islands"). The other vitrified groups (GS60s and GS100s) also demonstrated a similar morphology, but with an even smaller residual glaze remaining on the surface. The same pattern could be observed in the mapping and BSD images (Figure 2). Therefore, as the 10\% HF etching time increases, the amount of remaining glaze becomes scarcer.

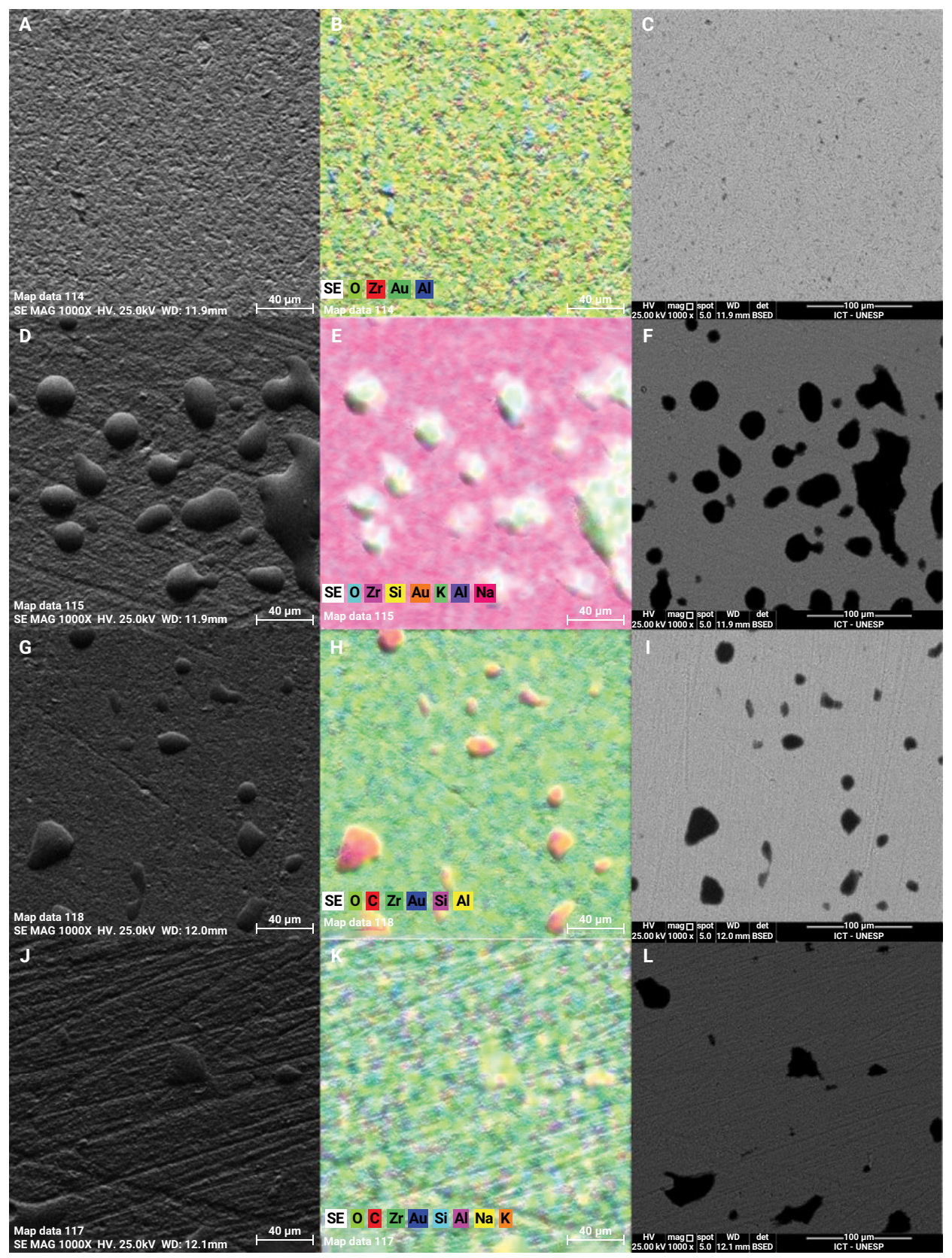

Figure 2. SEM, mapping and BSE (1000X) of the control group RS (A,B,C), showing homogenous surface; and the experimental GS20s (D,E,F), GS60s (G,H,I) and GS100s $(\mathrm{J}, \mathrm{K}, \mathrm{L})$, which reinforce the irregular distribution of the glass layer on the Y-TZP surface, forming the "glaze islands". 

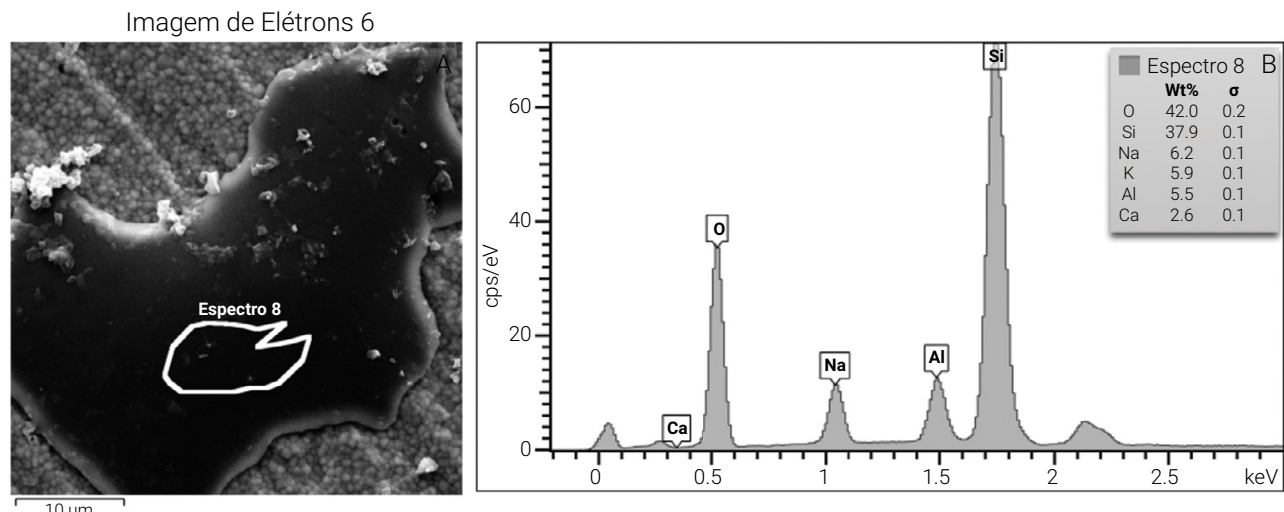

Figure 3. SEM $(20,000 x)$ of the GS60s group indicating the area analyzed by EDS (A) and the graphical representation of the EDS analysis indicating a considerable presence of silica (37.9\%) in one of the "glaze islands" (B).

EDS analysis showed the presence of the following elements in its composition: Aluminum (Al), Calcium (Ca), Potassium (K), Sodium (Na), Oxygen (O), Silica (Si) and Zirconia ( $\mathrm{Zr}$ ). The weight (\%) for each chemical element in the RS group was: Al (1.9\%), O (30.0\%), Si (1.8\%) and Zr (66.3\%). For GS20s: Al (1.3\%), K (1.7\%), Na (1.7\%) O (38.7\%), Si (6.6\%) and Zr (60.1\%). For GS60s: Al (0.4\%), O (24.5\%), Si (0.8\%) and $\operatorname{Zr}(74.3 \%)$. And finally for GS100s: Al (0.9\%), K (0.4\%), Na (0.5\%) O (25.3\%), Si (1.9\%) and $\operatorname{Zr}(71.4 \%)$.

In the extra GS60s sample, one more measurement of the chemical composition was performed specifically on an "glaze island" area (Figure 3). In this area, the weight (\%) for each chemical element was: Al (5.5\%), Ca (2.6\%), K (5.9\%), Na (6.2\%), O (42\%), and Si (37.9\%).

\section{DISCUSSION}

Previous studies showed that chemical and/or mechanical modification by the application of a thin glass layer in the Y-TZP ceramic surface positively influences bonding strength to resin cements ${ }^{3,19-20}$. As such, this research was carried out in order to evaluate the effect of three different HF etching times on the glaze surface of a Y-TZP ceramic associated with the use of a universal adhesive and resinous dual-cure cement.

The control group of this research was constituted by sandblasting with silica-coated alumina Y-TZP, with subsequent application of the universal adhesive containing a silane coupling agent ${ }^{3,15,23}$. This procedure promotes chemical adhesion between the ceramic surface and the resin cement organic matrix ${ }^{3}$. This happens by the attachment of silane monomers that react with the silica-coated surface within the silanol groups, thus forming hydrogen bonds and finally a covalently bonded very thin silane film ${ }^{6,12}$. Then, silane film with its free carbon-carbon double bonds reacts with the double bonds of resin composite luting cement ${ }^{12}$. Thus, good bond strength is obtained. An acceptable range of bond strength is 10 to $13 \mathrm{Mpa}^{24}$. In the present study, even after aging, the control group bond strength was $22 \mathrm{MPa}$. This high result may 
be related to the use of this surface treatment associated to an adhesive system with $\mathrm{MDP}^{1,6,10,21}$. The chemical interaction of this monomer can improve bond strength of crystalline ceramics such as zirconia, since this monomer has two bonding ends: one end has vinyl groups that react with the monomers of the resin cement when cured, and the other has phosphate ester groups which have strong hydrophilic bonding to metal oxides 2,21 .

Despite the promising results of bond strength using sandblasting with silica-coated alumina treatment, previous studies have demonstrated that this method can create a critical damage zone involving grooves and defects that can generate clinical failures $^{3,14}$. Because of this, an alternative approach was introduced to improve bond strength to Y-TZP ceramics and resin cement ${ }^{3,6,7}$. This other treatment involves a thin glass layer applied to the zirconia surface ${ }^{3,7}$. This enriches the surface with silicon oxides, which facilitates chemical bonding through the silane application. In turn, this produces a siloxane bond between the silica contained in this new vitrified layer and the resin cement organic matrix ${ }^{3,7}$. In addition, the vitrification allows HF etching of the glass layer, which modifies the surface topography and creates micromechanical retentions, similar to the vitreous ceramics mechanisms ${ }^{3,5,11,17}$.

However, denying the first null hypothesis, the bond strength values in the vitrified groups were significantly lower than the control group. The GS60s had bond strength values closer to those considered acceptable in the literature ${ }^{24}$, while GS20s and GS100s presented lower values. Nonetheless, as in a previous study on a naturally vitreous ceramic ${ }^{18}$, the HF etching time on the glass layer revealed no statistical difference in the bond strength results of the ceramic with a resinous cement, leading to the acceptance of the second null hypothesis. However, from the statistical point of view, the small number of samples per group in this research may be responsible for the high variation coefficient and caused a low power of the test, increasing the probability of a false negative result ${ }^{8}$.

Martins et al. ${ }^{5}$ stated that the amount of silica on the zirconia surface is higher when sandblasting with silica-coated alumina compared to vitrification, which could justify the obtained result; however, our EDS results do not corroborate this information, because the silica percentage on the different groups was similar. The most acceptable justification for this is that sandblasting was able to create surface irregularities by which the adhesive system and cement penetration occurred on the Y-TZP (20). Despite this, other authors claim that the vitrification technique is an advantageous surface treatment for zirconia as a whole ${ }^{25}$; it is easy to apply, has satisfactory cost-benefit, and does not induce damage to the ceramic ${ }^{5,11,14,25}$

The glaze layer remaining after HF etching was not uniform, which does not favor the chemical and mechanical union desired from vitrification, and can justify the results. Through these tests, it was found that $10 \%$ HF etching of the glazed surface irregularly removes a considerable part of the glaze applied to the surface of the $\mathrm{Y}$-TZP, leaving only "glaze islands" and large regions without vitreous content on the zirconia. This removal was proportional to the application time of HF. Therefore, it is suggested that adhesion in these specimens are more related to the MDP presence in the adhesive system ${ }^{22}$. There is a statement that in order to produce better bond strength results, this monomer must be present in both the cement and the 
adhesive $8,21,22$; however, the present adhesive system only had MDP in the adhesive composition, but not in the cement.

Six thousand thermal cycles was adopted in this research, which is a quantity also used by other researchers $3,12,16,23$. Some experimental group samples were lost during aging. Previous studies have reported reduced bond strength or premature failure due to thermocycling, even in specimens treated witch silica-coated alumina ${ }^{15}$. This is due to the combination of hydrolytic degradation, water diffusion into the interfacial layer and thermal irradiation during cycles ${ }^{3}$. Therefore, it is observed that Y-TZP adhesive interfaces are susceptible to aging ${ }^{23}$. However, it is known that even though zirconia cemented with MDP-containing adhesive systems reduces adhesion after thermocycling ${ }^{8}$, the presence of this phosphate monomer generates better conditions to support this aging ${ }^{26}$. This is due to the monomers' chemical bonding to the metal oxides by Van Der Waals forces or hydrogen bonds at the resin cement/zirconium interface ${ }^{10}$.

Failure analysis indicated that these were always adhesive, independently of the groups, and the zirconia blocks were adhesive and cement free. This has also been observed in other studies ${ }^{1,8,12,21,26}$. These failures may be associated with several factors: thermal expansion difference between the materials ${ }^{8,25}$, processing techniques, phase transformation and factors related to the adhesive system ${ }^{25}$. In the universal adhesive chemical composition there are the MDP, dimethacrylate, 2-hydroxyethyl methacrylate, vitrebond copolymer, ethanol, water, initiators and silane. However, mixing these constituents in the same flask containing a greater amount of solvents can hinder the adhesion between resin cement and ceramic, because they react differently in each substrate ${ }^{13}$. In addition, Kim et al. ${ }^{27}$ claimed that silane incorporation into the universal adhesive appears to be ineffective, and that the MDP may prevent optimal chemical interaction between silane and ceramic, which is due to the tendency for premature hydrolysis in an acidic environment. Some authors argue that systems containing metal primer and silane in separate flasks promote better chemical bonding $5,13,26$.

Lastly, through EDS of a "glaze island" area it was possible to observe the high presence of silica content (37.9\%) (Figure 3). In addition, the contact angle results showed that lower etching time results in a higher amount of glaze being maintained on the surface, higher wettability and better adhesion. Therefore, the application and more importantly the maintenance of this glass layer on the Y-TZP surface seems to be a promising path for zirconia adhesive luting. New protocols have yet to be evaluated, such as application of a double or triple glaze layer on a zirconia surface or the use of powder/liquid glaze by brush technique, in order to obtain better standardization of the glaze application, which does not seem to be guaranteed with the spray application.

In conclusion, the shear bond results demonstrated that the bond strength between Y-TZP glazed and an adhesive system with MDP was not influenced by different conditioning times with hydrofluoric acid. However, the image tests and goniometry indicate that a shorter HF etching time is more favorable for the adhesive surface of this zirconia. 


\section{REFERENCES}

1. Cavalcanti AN, Foxton RM, Watson TF, Oliveira MT, Giannini M, Marchi GM. Bond strength of resin cements to a zirconia ceramic with different surface treatments. Oper Dent. 2009 May-Jun;34(3):280-7. doi: 10.2341/08-80.

2. Özcan $M$, Bernasconi M. Adhesion to zirconia used for dental restorations: a systematic review and meta-analysis. J Adhes Dent. 2015 Feb;17(1):7-26. doi: 10.3290/j.jad.a33525.

3. Amaral M, Belli R, Cesar PF, Valandro LF, Petschelt A, Lohbauer U. The potential of novel primers and universal adhesives to bond to zirconia. J Dent. 2014 Jan;42(1):90-8. doi: 10.1016/j.jdent.2013.11.004.

4. Gargava S, Ram SM. Evaluation of Surface Conditioning of Zirconia and Its Effect on Bonding to Resin-Luting Agent. J Contemp Dent. 2013 Jan-Apr;3(1):7-10. doi: 10.5005/jp-jourals-10031-1026.

5. Martins ARM, Gotti VB, Shimano MM, Borges GA, Gonçalves LS. Improving adhesion between luting cement and zirconia-based ceramic with an alternative surface treatment. Braz Oral Res. 2015;29:54. doi: 10.1590/1807-3107BOR-2015.vol29.0054.

6. Pereira LL, Campos F, Dal Piva AM, Gondim LD, Souza RO, Özcan M. Can application of universal primers alone be a substitute for airborne-particle abrasion to improve adhesion of resin cement to zirconia? J Adhes Dent. 2015 Apr;17(2):169-74. doi: 10.3290/j.jad.a33974.

7. Ntala P, Chen X, Niggli J, Cattell M. Development and testing of multi-phase glazes for adhesive bonding to zirconia substrates. J Dent. 2010 Oct;38(10):773-81. doi: 10.1016/j.jdent.2010.06.008.

8. Zhao L, Jian YT, Wang XD, Zhao K. Bond strength of primer/cement systems to zirconia subjected to artificial aging. J Prosthet Dent. 2016 Nov;116(5):790-6. doi: 10.1016/j.prosdent.2016.03.020.

9. Attia A. Bond strength of three luting agents to zirconia ceramic - influence of surface treatment and thermocycling. J Appl Oral Sci. 2011 Aug;19(4):388-95.

10. Tanıs MT, Akay C, Karakıs D. Resin cementation of zirconia ceramics with different bonding agentes. Biotechnol Biotechnol Equip. 2015 Mar 4;29(2):363-7.

11. Vanderlei A, Bottino MA, Valandro LF. Evaluation of resin bond strength to yttria-stabilized tetragonal zirconia and framework marginal fit: comparison of different surface conditionings. Oper Dent. 2014 Jan-Feb;39(1):50-63. doi: 10.2341/12-269-L.

12. Özcan $M$, Yetkiner $E$. Could readily silanized silica particles substitute silica coating and silanization in conditioning zirconium dioxide for resin adhesion? J Adhes Sci Technol. 2016;30(2):186-93. doi: 10.1080/01694243.2015.1095628.

13. Alves M, Campos F, Bergoli CD, Bottino MA, Özcan M, Souza R. Effect of Adhesive Cementation Strategies on the Bonding of Y-TZP to Human Dentin. Oper Dent. 2016 May-Jun;41(3):276-83. doi: 10.2341/15-052-L.

14. Melo RM, Souza R, Dursun E, Monteiro E, Valandro LF, Bottino MA. Surface Treatments of Zirconia to Enhance Bonding Durability. Oper Dent. 2015 Nov-Dec;40(6):636-43. doi: 10.2341/14-144-L.

15. Usumez A, Hamdemirci N, Koroglu BY, Simsek I, Parlar O, Sari T. Bond strength of resin cement to zirconia ceramic with different surface treatments. Lasers Med Sci. 2013 Jan;28(1):259-66. doi: 10.1007/s10103-012-1136-x.

16. Rippe MP, Amaral R, Oliveira FS, Cesar PF, Scotti R, Valandro LF, et al. Evaluation of tensile retention of Y-TZP crowns cemented on resin composite cores: effect of the cement and Y-TZP surface conditioning. Oper Dent. 2015 Jan-Feb;40(1):E1-E10. doi: 10.2341/13-310-L.

17. Bottino MA, Snellaert A, Bergoli CD, Özcan M, Bottino MC, Valandro LF. Effect of ceramic etching protocols on resin bond strength to a feldspar ceramic. Oper Dent. 2015 Mar-Apr;40(2):E40-6. doi: 10.2341/13-344-L. 
18. Leite FP, Ozcan M, Valandro LF, Moreira CHC, Amaral R, Botino MA, et al. Effect of the etching duration and ultrasonic cleaning on microtensile bond strength between feldspathic ceramic and resin cement. J Adhes. 2013;89:159-73. doi: 10.1080/00218464.2013.739024.

19. Miranda JS, Malta NV, Carvalho RLA, Souza ROA, Machado JPB, Leite FPP. Which low-fusing porcelain glaze treatment technique is better to promote a vitreous surface on Y-TZP ceramic? Rev Odonto Cienc. 2017;32(4):174-9. doi: 10.15448/1980-6523.2017.4.28749.

20. Simões AC, Miranda JS, Souza ROA, Kimpara ET, Leite FPP. Bond and topography evaluation of a Y-TZP ceramic with a superficial low-fusing porcelain glass layer after different hydrofluoric acid etching protocols. Rev Odontol UNESP. 2018 Nov-Dec;47(6):1-6. doi: 10.1590/1807-2577.10118.

21. Gotti VB, Calabrez Filho S, Shimano MM, Borges GA, BorgeS LG, Gonçalves LS. Influence of ceramic primers on microshear bond strength between resin cements and zirconia-based ceramic. Braz J Oral Sci. 2011 Apr-Jun;10(2):124-9.

22. Ahn J, Yi Y, Lee Y, Seo D. Shear Bond Strength of MDP-Containing Self-Adhesive Resin Cement and Y-TZP Ceramics: Effect of Phosphate Monomer-Containing Primers. Biomed Res Int. 2015;2015:389234. doi: 10.1155/2015/389234.

23. Sarmento HR, Campos F, Sousa RS, Machado JP, Souza RO, Bottino MA, et al. Influence of air-particle deposition protocols on the surface topography and adhesion of resin cement to zirconia. Acta Odontol Scand. 2014 Jul;72(5):346-53. doi: 10.3109/00016357.2013.837958.

24. Lüthy $\mathrm{H}$, Loeffel $\mathrm{O}$, Hammerle $\mathrm{CH}$. Effect of thermocycling on bond strength of luting cements to zirconia ceramic. Dent Mater. 2006 Feb;22(2):195-200.

25. Anami LC, Lima JM, Corazza PH, Yamamoto ET, Bottino MA, Borges AL. Finite element analysis of the influence of geometry and design of zirconia crowns on stress distribution. J Prosthodont. 2015 Feb;24(2):146-51. doi: 10.1111/jopr.12175.

26. Kim MJ, Kim YK, Kim KH, Kwon TY. Shear bond strengths of various luting cements to zirconia ceramic: surface chemical aspects. J Dent. 2011 Nov;39(11):795-803. doi: 10.1016/j.jdent.2011.08.012.

27. Kim RJY, Woo JS, Lee IB, Yi YA, Hwang JY, Seo DG. Performance of universal adhesives on bonding to leucite-reinforced ceramic. Biomater Res. 2015 May 22;19:11. doi: 10.1186/s40824-015-0035-1. 